Published as: Youssef, R. (2021). Abweichung und Konformität: Prolegomena zu einer historischen Wissenssoziologie der Imitation. In Grünbart, Michael, Schwedler, Gerald \& Sonntag, Jörg (eds.), Imitationen: Systematische Zugänge zu einem kulturellen Prinzip des Mittelalters (Bd. 83, S. 29-49). Paderborn: Brill | Fink.

https://doi.org/10.30965/9783846764183_003

\title{
Abweichung und Konformität - Prolegomena zu einer historischen Wissenssoziologie der Imitation
}

\section{Ramy Youssef}

\section{Imitation als Gegenstand soziologischer und interdisziplinärer Forschung}

Imitation ist in der Soziologie wieder zum Thema grundlagentheoretischer Forschung geworden ${ }^{1}$. In diesem , wieder' verbirgt sich eine ungewöhnlich lange Flaute, die zwischen der Gründerzeit und der Gegenwart soziologischer Forschung zu beobachten ist. In den Anfängen der Disziplin ragt freilich das Werk Gabriel de Tardes als nach wie vor ambitioniertester Versuch heraus, die Soziologie als W i s s e n s c h a f t d e r I m it a ti on schlechthin zu begründen² ${ }^{2}$ Beinahe schien es so, als ob mit dem Theorem der Imitation die drängendsten soziologischen Fragen geklärt werden könnten: das Verhältnis zwischen Individuum und Gesellschaft, zwischen Differenzierung und Integration, zwischen Konsens und Konflikt und schließlich zwischen Stabilität und sozialem Wandel. Dass es dennoch nicht zur nachhaltigen Weiterentwicklung dieses Forschungsprogramms kam, wird üblicherweise weniger dem Werk Tardes selbst als vielmehr dem alles andere überstrahlenden Erfolg der Soziologie Émile Durkheims zugerechnet ${ }^{3}$. Das bedeutet nicht, dass die Leistung Tardes völlig in Vergessenheit geraten wäre. Aber mit seinen ,Lois de l'imitation' schien bereits alles zum Thema gesagt worden zu sein. Selbst ein innovativer Soziologe wie Georg Simmel nahm zwar die Nachahmung neben anderen sozialen Formen wie Konkurrenz, Konflikt oder Über- und Unterordnung in den Katalog seiner sozialen Formenlehre auf, führte sie aber kaum näher aus, sondern schrieb stattdessen in einer Rezension Tarde das Verdienst zu, die Form der

\footnotetext{
${ }^{1}$ Christian BorCH - UrS StÄHEli (Hgg.), Soziologie der Nachahmung und des Begehrens. Materialien zu Gabriel Tarde, Frankfurt/Main 2009; SUSAN L. HuRLEY - NICK CHATER (Hgg.), Perspectives on imitation. From neuroscience to social science, Cambridge, Mass 2005; SERGIO TONKONOFF, A new social physic. The sociology of Gabriel Tarde and its legacy, in: Current Sociology 61, 2013, S. 267-282.

${ }^{2}$ GABRIEL DE TARDE, Les lois de l'imitation. Étude sociologique, Paris 1890.

3 Roger L. Geiger, The institutionalization of sociological paradigms. Three examples from early French sociology, in: Journal of the History of the Behavioral Sciences 11, 1975, S. 235-245; BJøRN THOMASSEN, Émile Durkheim between Gabriel Tarde and Arnold van Gennep. Founding moments of sociology and anthropology, in: Social Anthropology 20, 2012, S. 231-249.
} 
Nachahmung herausgearbeitet zu haben ${ }^{4}$. Das gegenwärtige soziologische Revival des Themas ist indes den Bemühungen Bruno Latours ${ }^{5} \mathrm{zu}$ verdanken und im Falle des deutschsprachigen Raumes vor allem der erstmaligen Übersetzung der ,Lois de l'imitation' ins Deutsche, welcher übrigens ein Begleitwort Latours vorangestellt wurde 6 .

In der Geschichtswissenschaft dürfte das Interesse an Imitation einem allgemeinen Trend zu kulturwissenschaftlichen Fragestellungen zu verdanken sein, der nicht zuletzt zu einer Annäherung zwischen Soziologie und Geschichtswissenschaft beigetragen hat. Diese erfolgt aber nicht ohne Hürden und Ambivalenzen. Denn während die Geschichtswissenschaft bereitwillig und kompetent soziologische Theoreme operationalisiert, verhält sich die Soziologie noch relativ reserviert gegenüber historischen Fragestellungen. Obwohl die Rufe nach einer historischen Soziologie lauter werden ${ }^{7}$, führt sie immer noch eine randständige Existenz und lässt ihr Senkblei selten tiefer als bis zur Frühen Neuzeit hinab in den Brunnen der Vergangenheit. Außerdem kann sie schon wegen ihres eigenen disziplinär und curricular bedingten Lektüreverhaltens nicht mit umfassenden Quellenkenntnissen aufwarten und ist deshalb sowohl auf Zufallsfunde als auch auf Kooperation mit der Geschichtswissenschaft angewiesen ${ }^{8}$. Um in diesem Sinne heterogene disziplininterne und interdisziplinäre Forschungsstränge produktiv miteinander ins Gespräch $\mathrm{zu}$ bringen, scheint eine vergleichende Methode besonders erfolgversprechend zu sein. Denn erst der Vergleich zwingt zur Abstraktion von Problemen, die jenseits von Einzelfällen und historischen Kontexten wiedererkennbar bleiben müssen und damit die Übersetzung von Forschungsergebnissen in andere Kontexte erleichtern. Trifft dies zu, so kann man im Werk Niklas Luhmanns ein instruktives Vorbild dafür finden, wie aus Vergleichen interdisziplinäre Forschungsfragen generiert und bearbeitet werden können ${ }^{9}$.

Von dieser Vorlage ausgehend werden Vergleiche vor allem dann fruchtbar sein, wenn in der soziologischen und geschichtswissenschaftlichen Forschung schärfer

\footnotetext{
${ }^{4}$ Georg Simmel, Das Wesen der Materie nach Kant's Physischer Monadologie, Abhandlungen 1882-1884, Rezensionen 1883-1901, Frankfurt/Main 2000, S. 248-250. Siehe auch HARTMANN TYRELL, Einleitung, in: DERS. - OtThein RAmmstedt u. a. (Hgg.), Georg Simmels große ,Soziologie‘. Eine kritische Sichtung nach hundert Jahren, Bielefeld 2011, S. 9-68, hier S. 48.

${ }^{5}$ BRUnO Latour, Gabriel Tarde und das Ende des Sozialen, in: Soziale Welt 52, 2001, S. 361-375.

${ }^{6}$ GABRIEL DE TARDE, Die Gesetze der Nachahmung, Frankfurt/Main 2003.

${ }^{7}$ RAINER SCHÜTZEICHEL, Historische Soziologie, Bielefeld 2004.

${ }^{8}$ NiKlas LUHMANN, Ansprüche an Historische Soziologie, in: Soziologische Revue 17, 1994, S. $259-264$.

${ }^{9}$ FrANK BECKER (Hg.), Geschichte und Systemtheorie. Exemplarische Fallstudien, Frankfurt/Main - New York 2004; Rudolf Stichweh, Systemtheorie und Geschichte, in: FranK WelZ - UWE WeISENBACHER (Hgg.), Soziologische Theorie und Geschichte, Wiesbaden 1998, S. 68-79.
} 
zwischen indigenen und analytischen Begriffen, zwischen Kategorien der Theorie und Kategorien der Praxis unterschieden wird. Denn ehe sie zum Gegenstand der Forschung werden, haben Praktiken des Imitierens immer schon Beschreibungen ihrer selbst angefertigt, deren Begrifflichkeit historisch variiert. In dem Maße, wie es der Forschung gelingt, ihre eigene Begrifflichkeit von diesen Vorgaben des Gegenstandes zu emanzipieren, kann sie nicht nur die konstitutive Funktion solcher Selbstbeschreibungen beleuchten, sondern auch historischen Wandel anhand dieser Selbstbeschreibungen nachvollziehen ${ }^{10}$. Hierfür kann eine Wissenssoziologie, die zwischen Selbstbeschreibung und (soziologischer bzw. geschichtswissenschaftlicher) Fremdbeschreibung differenziert $^{11}$, auf mindestens drei bewährte funktional äquivalente Techniken der Distanzierung und ,Verfremdung' ihres Gegenstandes zurückgreifen: die konstruktivistische Deutung, die funktionale Analyse und die Historisierung von Selbstbeschreibungen. Für eine soziologische Beschreibung des Imitierens sollen hier alle drei Techniken synthetisiert werden, um einen Begriff zu entwickeln, der I m itati on als kommunizierte Anwendung eines semantisch konstituierten Schemas auffasst, das bestimmte Probleme der Beobachtung und Kontrolle von Abweichungen lösen kann. Diese funktionale und damit auch ahistorische Definition soll in den folgenden Abschnitten begründet und im Lichte historisch variabler Rahmenbedingungen betrachtet werden.

\section{Imitation als semantisches Artefakt}

In einer konstruktivistischen Perspektive ist Imitation zuallererst als eine Praxis aufzufassen, die sich selbst als Imitation beschreibt und konstruiert. Erst die kommunikative Sinnbestimmung einer Handlung als Nachahmung konstituiert das Imitieren als einen sozialen Tatbestand ${ }^{12}$. Nur wenn von dieser Prämisse ausgegangen wird, kann Imitation von anderen Praktiken unterschieden werden, die verwechselbare Effekte der Ähnlichkeit, Vervielfältigung oder Diffusion zeitigen. Und nur auf diese Weise kann verhindert werden, dass die Forschung Kategorien über einen Gegenstand stülpt, die ihm nicht gerecht werden.

\footnotetext{
${ }^{10}$ NiKLAS LUHMANN, Ideenevolution. Beiträge zur Wissenssoziologie, Frankfurt/Main 2008.

11 ANDRÉ KIESERLING, Selbstbeschreibung und Fremdbeschreibung. Beiträge zur Soziologie soziologischen Wissens, Frankfurt/Main 2004.

${ }^{12}$ Siehe für eine handlungstheoretische Formulierung MAX WEBER, Wirtschaft und Gesellschaft, Tübingen 1922 , S. 11-12.
} 
Bereits hier kann die Forschung aber auf erhebliche Hürden stoßen, denn Selbstbeschreibungen der Imitation findet man nicht immer in der Form geschriebener Geständnisse vor, sondern müssen oft mühselig aus Artefakten der ,material culture' oder der ikonischen Semantik rekonstruiert werden. Hat man es indes mit einer Gesellschaft zu tun, die außerdem über Schrift verfügt, kann die Analyse ihrer begrifflichen Semantik Aufschluss darüber geben, ob es überhaupt ein ausgeprägtes gesellschaftliches B e wus st se in für Probleme des Imitierens gibt. Das trifft auf orale Kulturen womöglich nur bedingt zu, in denen sich Kommunikation beinahe ausschließlich unter räumlich ko-präsenten Anwesenden vollzieht, etwa wenn die Nachahmung (z. B. von rhythmischen Bewegungen in einem Ritual) beinahe simultan mit dem Vorbild zu erfolgen hat. Demgegenüber scheint erst die Einführung von Schrift nicht nur Kommunikation schlechthin, sondern ebenso Probleme des Imitierens im Besonderen beobachtbar zu machen ${ }^{13}$. Denn die Differenz zwischen einem Vorbild und dessen Nachahmung ist vor allem dann sinnfällig, wenn beide nicht mehr demselben unmittelbaren Wahrnehmungskontext angehören müssen, sondern zeitlich und sachlich (etwa durch Abstraktion des Vorbildes) voneinander deutlich unterscheidbar werden und die Beobachtung solcher Differenzen nicht von unmittelbaren Handlungszwängen belastet ist. Schrift steigert im Vergleich zur Kommunikation unter Anwesenden beide Leistungen wechselseitig aneinander ${ }^{14}$ : In zeitlicher und sachlicher Hinsicht erhöht Schriftlichkeit kommunikative Redundanz, da sie sich vervielfältigen und zeitlich fixieren lässt. Ferner erlaubt sie, zwischen überlieferter Schrift und gegenwärtiger Interpretation (oder Rezeption) zu unterscheiden und mit großzügigerem Zeitbudget auf Ähnlichkeiten und Abweichungen hin $\mathrm{zu}$ kontrollieren. Und schließlich begünstigt sie die Ausdifferenzierung von handlungsentlasteten Beobachterrollen (Philosophen), die mit der Pflege einer anspruchsvollen und abstrakten Semantik befasst sind. Damit dürfte gerade die Schrift zumindest im Falle der alteuropäischen Semantik erste Überlegungen zur Imitation ermöglicht haben, die mit Begriffen wie mimesis oder imitatio thematisiert werden.

Erst derartige Semantiken konstituieren in der nachträglichen Beobachtung einer Handlung, einer Praxis oder eines beliebigen Sinngehaltes Imitation als sozial folgenreiches Phänomen. Sie wird philosophisch anspruchsvoll und unter erhöhten

\footnotetext{
${ }^{13}$ CORNELIA BOHN, Schriftlichkeit und Gesellschaft. Kommunikation und Sozialität der Neuzeit, Wiesbaden 1999 , S. $134-135$.

${ }^{14}$ Ebd., S. 67-69; NiKLAS LuHMANN, Die Gesellschaft der Gesellschaft, Frankfurt/Main 1997, S. $249-251$.
} 
begrifflichen Konsistenzanforderungen ,gepflegt', kann sich aber auch auf der Ebene sozialer Praxis artikulieren, wo es gleichwohl mehr auf alltagsweltliche Plausibilität als auf semantische Feinheiten ankommt. Soziale Strukturen und Semantiken variieren jedenfalls nicht unabhängig voneinander, wie später noch zu zeigen sein wird. Zuvor muss jedoch geklärt werden, welches Problem mit Semantiken der Imitation gelöst wird und welche möglichen Alternativen denkbar wären.

\section{Zur Funktion des Imitierens}

Wie oben bereits angemerkt wurde, markiert sich Imitation als soziales Phänomen durch Verwendung bestimmter Semantiken bereits selbst. Diese Semantiken variieren aber sowohl sprachlich als auch in ihren Konnotationen und Bedeutungsgehalten. Um dennoch sinnvoll vergleichen zu können, bietet sich die Angabe einer gemeinsamen Funktion an, die sich inkongruent $\mathrm{zu}$ variierenden Semantiken der Imitation verhält. Eine Funktionsangabe, die sich in diesem Sinne von Selbstbeschreibungen der Imitation distanziert, kann als ahistorisch definiertes ,tertium comparationis' konstant gehalten werden, um auf diese Weise diachrone und synchrone Vergleiche zu ermöglichen ${ }^{15}$. Der hier zu unterbreitende Vorschlag geht davon aus, dass der Schematismus der Imitation das Problem der Abweichungskontrolle löst, indem er eine Situation nach möglichen Spielräumen der Konformität und der Abweichung anhand von Ähnlichkeiten abtastet und dadurch soziales Verhalten orientiert.

Im Vollzug von Kommunikation sind Abweichungen jedenfalls unausweichlich. Schon auf einer zeitlichen Ebene ergeben sie sich dadurch, dass sich jede Kommunikation in Form von Ereignissen vollzieht, die sich im Augenblick ihres Erscheinens wieder verflüchtigen ${ }^{16}$. Während Ereignisse selbst unwiederholbar sind, lässt sich dafür ihr Sinngehalt reproduzieren - dies indes nur unter Inkaufnahme einer Sinnverschiebung bzw. ,différance' einerseits und eines neuen Ereignisses andererseits: Man kann in einer (etwas zum Erliegen gekommenen) Konversation darauf hinweisen, dass es regnet. Diese Bemerkung verfliegt, kaum, dass sie ausgesprochen wurde. Sie allein vermag eine Konversation nicht $\mathrm{zu}$ tragen und muss durch weitere Anschlussereignisse ersetzt werden. In diesem Fall wird es darauf ankommen, ob man den Sinn der vorangegangenen

\footnotetext{
${ }^{15}$ DERS., Funktionale Methode und Systemtheorie, in: Soziologische Aufklärung, Bd. 1: Aufsätze zur Theorie sozialer Systeme, Opladen 1974, S. 31-53.

16 Niklas LuhmanN, Soziale Systeme, Frankfurt/Main 1984, S. 472-473; Ramy Youssef, On the Social Constitution of Structures, Actions and Events, in: InterDisciplines 7, 2016, S. 5-31.
} 
Bemerkung (diesmal nun: als Wiederholung!) wieder ins Spiel bringt und damit die Kommunikation in die Krise stürzt, oder ob neuer Sinn eingebracht werden kann. Ein Sinngehalt kann auch vor dem Hintergrund unterschiedlicher Perspektiven, ungleich verteilten Wissens und individuell unterschiedlicher Aufmerksamkeitsschranken als Abweichung, Überraschung oder Enttäuschung auffallen. Ein anwesender Meteorologe wird die Mitteilung, dass es regnet, vor dem Hintergrund des aktuellen Wetterberichts auffassen, während einem anwesenden Soziologen bei diesem Anlass etwa Erving Goffmans berühmtes Diktum einfallen kann: „[C]onversation can burn everything.“17 Damit ist gemeint, dass Kommunikation gerade unter Anwesenden, die einander wechselseitig wahrnehmen, ein gewisses Maß an Aufmerksamkeit einmahnt, sich dadurch in ständige Unruhe und ,Kollektivnervosität'18 versetzt und sensibel für Irritationen und Störungen wird, die als Kommunikationsbeiträge verwertet werden können - wie banal diese auch immer sein mögen. Sie findet aber bei aller Anfälligkeit für Abweichungen, Neuigkeiten und Informationen ihren Gegenhalt in Redundanzen und Differenzen ${ }^{19}$. Kommunikative Ereignisse fallen mithin nur dann auf, wenn sie einen Informationswert haben, der sie von einem redundanten Hintergrund abhebt. Jedes sinnhafte Erleben ist immer schon von Redundanzen in der Form relativ invariabler Typisierungen angeleitet ${ }^{20}$; man denke nur an den Orientierungs- und Wiedererkennungswert des Interaktionstypus ,Warteschlange'. So kann man als Neuling an einer Universität die Erwartung bilden, dass in der hiesigen Mensa jenes Menü am besten schmeckt, für das sich die meisten Personen in der Schlange anstellen und sie dann nachahmen. Der Verlass auf derartige Redundanzen kann sich jedoch als fatale (kulinarische) Fehleinschätzung entpuppen. An dieser Stelle können Differenzen vor allem in der Form von Alarmsignalen und Widersprüchen zur Selbstkorrektur der Kommunikation eingesetzt werden: Man bemerkt, dass sich die längsten Schlangen regelmäßig vor dem vegetarischen Menü bilden, und man wird mit einer Bourdieuschen Optik milieuspezifische und moralisch sanktionierbare Diäterwartungen feststellen können, denen man nicht ohne weiteres nachgeben möchte. Zudem bedarf es mitunter

\footnotetext{
${ }^{17}$ Erving Goffman, Forms of talk, Philadelphia 1981, S. 38.

${ }^{18}$ GEORG SIMMEL, Über sociale Differenzierung. Sociologische und psychologische Untersuchungen, Leipzig 1890, S. 82.

${ }^{19}$ LUHMANN, Soziale Systeme (wie Anm. 16), S. 237.

${ }^{20}$ ALFRED SCHÜTZ, Der sinnhafte Aufbau der sozialen Welt. Eine Einleitung in die verstehende Soziologie, Wien 1932.
} 
einer gehörigen Portion Ich-Stärke, um dem moralischen Konformitätsdruck und kollektiven Naserümpfen standzuhalten und sich vor der Grilltheke anzustellen.

Es gibt also bewährte Redundanzen, die zu allgemein erwartbaren Erwartungen bzw. zu sozialen Strukturen gerinnen können, denen erwartungsgemäß entsprochen werden kann. Kommunikation bietet erst vor diesem Hintergrund laufend Anlässe für Überraschungen und Enttäuschungen, wobei diese durch Alarmvorrichtungen sozial sichtbar gemacht werden müssen, und dann die Frage aufwerfen, ob im Enttäuschungsfall an sozialen Erwartungen normativ festgehalten wird oder ob sie an eine neue Sachlage anzupassen sind. Da jedoch Ereignisse (und Speisepläne) ständig variieren, können sie nicht im Vorhinein exakt antizipiert werden, sondern müssen generalisiert und typisiert werden. Soziale Strukturen eröffnen damit zugleich jene Spielräume für Variabilität und Redundanz, für Konformität und für Abweichung, in denen man auf Imitation zurückgreifen kann, um sich zu orientieren.

Eine Orientierung bietet der Schematismus der Imitation zunächst durch seine Ausrichtung an zeitlich vorgelagerten Sinngehalten, also: an Vorbildern im weitesten Sinne. Er misst mit dem sachlichen Index der Ähnlichkeit ${ }^{21}$ Abweichung und Konformität sozialen Verhaltens, zeigt an, wo man auf halbwegs sicheren Pfaden wandelt, und erzeugt semantische Alarmsignale, wo Grenzwerte sozial erwartbaren Verhaltens überschritten werden. Ein mögliches Alarmsignal kann bereits die Thematisierung von Imitation darstellen, wenn etwa ein bestimmtes Verhalten, das man als Ausdruck seiner eigenen Persönlichkeit und Individualität empfunden hat, plötzlich als versuchte Imitation einer fremden Person ausgelegt wird und damit die Gewissheit der eigenen Identität ins Wanken gerät. In Grenzfällen der ,Verähnlichung‘ kann mit Semantiken der Imitation auf die Möglichkeit der Verwechslung, wenn nicht sogar der Substitution aufmerksam gemacht werden. Denn wer es mit der imitatio Christi zu weit treibt, könnte sowohl unter Anhängern als auch unter Widersachern den Verdacht erwecken, sich selbst für göttlich zu halten.

\footnotetext{
${ }^{21}$ Matthias Junge, Identifikation durch mimetische Imagination und die Bedeutung von Ähnlichkeit für die Vergesellschaftung, in: Winfried GEBHARDT - RonAld Hitzler (Hgg.), Nomaden, Flaneure, Vagabunden. Wissensformen und Denkstile der Gegenwart, Wiesbaden 2006, S. 84-99.
} 
Um eine soziale Orientierung zu ermöglichen, verknüpft Imitation außerdem bestimmte Leistungen in drei Dimensionen sozialen Sinns ${ }^{22}$ und steigert sie wechselseitig aneinander:

In der Z e itdimen sion ermöglicht Imitation eine Orientierung an vorherigen und womöglich bereits bewährten Problemlösungen, deren künftige Erfolgsaussichten und Folgeprobleme zu einem gewissen Ausmaß antizipiert werden können. Man muss das Rad nicht ständig neu erfinden, spart dadurch Zeit, kann auf Erfahrungswerte verweisen und diese in eine dadurch weniger riskant erscheinende Zukunft extrapolieren. Ein Vorbild wird dann als Ziel definiert, sodass Imitation in eine Aufeinanderfolge mehrerer Etappen sequenzialisiert wird, deren Bewältigung ebenso etappenweise bis zur Zielerreichung mit einem Ähnlichkeitsindex laufend kontrolliert werden kann: Ein neuer Tanz wird buchstäblich schrittweise erlernt, indem die entsprechenden Schritte vorgeführt und nachgeahmt werden, wobei auftretende Fehler wiederum sichtbar gemacht und korrigiert werden können, indem der Lehrer die Fehler des Schülers nachahmt und vorführt ${ }^{23}$. Das Beispiel deutet bereits an, dass die dabei relevanten Zeithorizonte häufig kaum über eine aktuelle Gegenwart hinausweisen müssen, sondern dass die Abstimmung zwischen Vorlage und Nachahmung etwa unter Bedingungen der Kopräsenz beinahe simultan erfolgen kann. Dies scheint insbesondere auf die Imitation von Körperpraktiken zuzutreffen, wenn es gilt, die Ähnlichkeit von Körperbewegungen auf Abweichung und Übereinstimmung hin zu kontrollieren.

Anders verhält es sich, wenn nicht Ereignisse, sondern Objekte imitiert werden. Ein Unternehmen kann sowohl versuchen, ein billigeres Imitat eines erfolgreichen Produktes herzustellen, als auch Produkte mit vergleichbarer Qualität auf den Markt zu bringen, indem der Herstellungsprozess am Konkurrenzprodukt selbst rekonstruiert und nachgeahmt wird. Vor diesem Hintergrund leuchtet es ein, dass gerade ein solches Prozesswissen vor Nachahmern geschützt wird, während die Zirkulation des Produktwissens mit den Produkten selbst unvermeidbar einhergeht ${ }^{24}$. Mit ganz anderen Zeithorizonten sind hingegen Historiker und Archäologen konfrontiert, die Imitation rückwirkend vom Ergebnis aus betrachten und rekonstruieren müssen und häufig nur

\footnotetext{
${ }^{22} \mathrm{Zu}$ den drei Sinndimensionen siehe LuHMANN, Soziale Systeme (wie Anm. 16), S. 111-113.

${ }^{23}$ LeElo KeEvallik, Bodily Quoting in Dance Correction, in: Research on Language \& Social Interaction 43 , 2010, S. 401-426.

${ }^{24}$ ANTOINETTE WeIBEL, Kooperation in strategischen Wissensnetzwerken. Vertrauen und Kontrolle zur Lösung des sozialen Dilemmas, Wiesbaden 2004, S. 52-63.
} 
aus der Ähnlichkeit und Datierung von Artefakten auf kulturelle Traditions- und Translationszusammenhänge schließen und diese voneinander abgrenzen können ${ }^{25}$.

Derartiger Fälle legen nahe, einen Zusammenhang zwischen Imitation und sozialem Gedächtnis zu vermuten. Ohne dies hier näher ausführen zu können, spricht einiges dafür, dass Redundanzen, die für den Aufbau eines sozialen Gedächtnisses unerlässlich sind, nicht durch Imitation erzeugt werden, sondern umgekehrt Imitation ein soziales Gedächtnis voraussetzt, das es ermöglicht, bewährte redundante Formen zu erinnern und deren Hintergrund zu vergessen. ${ }^{26}$ So setzt die imitatio Christi voraus, dass man sich an Leben und Taten Christi erinnert, entlastet aber insofern, als jene Glaubenslehren vergessen werden können, gegen die sich das Christentum historisch durchgesetzt hat. Und wer die Rhetorik Ciceros imitiert, kann dies getrost in Unkenntnis jener in Vergessenheit geratenen Zeitgenossen tun, mit denen sich Cicero auseinandersetzen musste.

In der Sachdimension, in der Objekte sinnhaft bestimmt und voneinander unterschieden werden, orientiert die Imitation eigene Handlungen nicht an vorangegangenen eigenen Handlungen oder Handlungsmustern, sondern an externen Formen. Man kann sich zwar unter Umständen selbst imitieren, muss sich dazu aber in eine Außenperspektive hineinversetzen, sich also selbst als einen anderen betrachten, und wird typischerweise bestimmte eigene auffällige Verhaltensweisen etwa in Form einer Selbstparodie einseitig steigern oder die Absicht der Imitation mitkommunizieren, um sie als solche überhaupt kenntlich zu machen. Sieht man von derartigen Fällen ab, ist die Regel also die Bezugnahme auf erlebte äußere Formen, die eigenes Handeln anleiten. Kontrolliert wird diese Referenz am Kriterium der Ähnlichkeit bzw. der Analogie. Die Vorteile dieses Kriteriums liegen einerseits darin, dass es auf Wahrnehmung zugreifen kann, die sehr schnell und unmittelbar eigene Zustände mit dem Vorbild abgleichen kann, und andererseits im intuitiv nachvollziehbaren Zusammenhang zwischen Vorbild und Nachahmung. Diese Vorteile müssen freilich mit der Einschränkung des Vergleichshorizontes auf tendenziell gleichartige Vorbilder finanziert werden: Soziologen orientieren sich an Soziologen, Künstler an Künstlern usw. Und wenn es doch zur Orientierung an unähnlichen Vorbildern kommt, so müssen höhere

\footnotetext{
${ }^{25}$ FABIO BARRY, Disiecta membra. Ranieri Zeno, the Imitation of Constantinople, the Spolia style, and Justice at San Marco, in: Henry Maguire - Robert Nelson (Hgg.), San Marco, Byzantium, and the Myths of Venice, Washington D.C. 2010, S. 7-62; FINBARR FLOOD, Objects of Translation. Material Culture and Medieval ,HinduMuslim' Encounter, Princeton 2009, S. 61-63.

${ }^{26}$ LUHMANN, Die Gesellschaft (wie Anm. 14), S. 581-583.
} 
Übersetzungsschwellen überwunden werden. Künstler können dann durch einen soziologischen Blick auffallen, während Soziologen Vorbilder in Lyrik und Poesie suchen ${ }^{27}$. Davon unberührt bleibt indes der wissenschaftliche Realitätsbezug der Soziologie, die sich dahingehend von der Kunst unterscheidet. Im Gegensatz zu konstitutiven und nicht-substituierbaren Funktionen bilden daher vermutlich übersetzbare ,Formen' den Gegenstand des Imitierens.

Formen sind immer zugleich Elemente, die in einem Komplex mit weiteren Elementen verknüpft sind. Im Sinne einer ,Gestalt' erregen schließlich nur einzelne Elemente Aufmerksamkeit und werden zum Gegenstand von Imitation, nicht aber die Kontexte und Relationen, die diese Elemente $\mathrm{zu}$ einem abgrenzbaren Komplex zusammenfassen ${ }^{28}$. Das Idol wird gemeinhin von seinen Taten, der Künstler von seinem Werk und ein Unternehmen von seinen Innovationen unterschieden. Im Rahmen der imitatio Christi kann zwar die Lebensführung Christi als wiedererkennbares Element eines ganzen Komplexes ,Christus' nachgeahmt und zur Grundlage eigenen Handelns werden. Die Funktion und die Stellung des Vorbilds Christus innerhalb der Heilsgeschichte ist jedoch nicht imitierbar ${ }^{29}$. Man kann - wenn man es kann! - die sfumato-Technik Leonardo da Vincis nachahmen, aber nicht ihren Stellenwert in der Beziehung $\mathrm{zu}$ anderen Elementen im Gesamtwerk des Künstlers oder in der Kunstgeschichte schlechthin. Mit jeder Anwendung des Imitationsschemas, mit jeder Übersetzung von fremden Formen werden daher immer Ambivalenzen und Widersprüche beobachtbar, die sich aus der Unübertragbarkeit von Kontexten und Relationen ergeben und die zur Proliferation weiterer Differenzen führen. Diese können (siehe Tarde!) selbst durch exzessive Nachahmung nicht eingeholt werden - auch wenn Elvis-Imitatoren eifrig und mit mehr oder weniger überzeugenden Ergebnissen daran arbeiten, ihr verstorbenes Idol am Leben zu erhalten. Differenzen dieser Art werden in der Praxis des Imitierens gerne übersehen und mitunter $\mathrm{zu}$ einer Einheit zusammengezogen. In der Analyse machen sie einen erheblichen Unterschied und

\footnotetext{
${ }^{27}$ ANDREw ABBotT, Against Narrative. A Preface to Lyrical Sociology, in: Sociological Theory 25, 2007, S. 6799.

${ }^{28}$ Alois HaHN, Aufmerksamkeit, in: DERS., Körper und Gedächtnis, Wiesbaden 2010, S. 63-96, hier S. 70-71.

${ }^{29}$ Das bezieht sich insbesondere auf die heilsgeschichtlich singulären Funktionen der jungfräulichen Geburt und des Todes Christi pro nobis, die weder imitiert noch substituiert werden können: Hunc in eo quisque sequitur, in quo imitator; non in quantum ille filius dei est unus per quem facta sunt omnia, sed in quantum filius hominis quae oportebat in se praebuit imitanda. Et multa in illo ad imitandum omnibus praeponuntur; uirginitas autem carnis non omnibus; non enim habent quid faciant ut uirgines sint, in quibus iam factum est ut uirgines non sint. Augustinus, De sancta virginitate, hg. von PATRICK G. WALSH, Oxford 2001, S. 66-147, hier S. 102.
} 
drängen sich in der Praxis spätestens dann auf, wenn der Kontext eines Vorbildes auf Imitationsversuche reagiert.

Treffen diese Vorbemerkungen zu, spannt sich die Praxis des Imitierens in einer T r i a d e zwischen dem Vorbild, der Nachahmungshandlung und einem weiteren Kontext auf. Das ist insofern kontraintuitiv, als dass Imitieren üblicherweise als eine Zweierbeziehung zwischen Vorbild und Nachahmung vorgestellt und die Imitation nur der Handlung des Nachahmers zugerechnet wird ${ }^{30}$. Übersehen wird dabei der Kontext des Vorbildes, zu welchem insbesondere der Urheber eines Vorbildes gehören kann, der schließlich nicht selbst und insgesamt, sondern nur in Ausschnitten imitiert wird. Imitiert man ein bestimmtes Element eines namhaften Künstlers, etwa ein populäres Lied, wird man daher mit Post von den Anwälten des Komponisten zu rechnen haben. Zum Kontext der Imitation gehört ebenso ein kritisches Publikum, welches eine Handlung im Schema der Imitation nach Gesichtspunkten der Abweichung oder Konformität, der Originalität oder der Formentreue beurteilt. Ahmt man die Stilmittel der klassischen auctores nach, können sich jene zwar nicht mehr selbst wehren, werden dafür aber von einem kritischen Publikum vertreten, das die Integrität der Klassiker zu bewahren trachtet - und zwar unabhängig davon, ob beim vermeintlichen Nachahmer eine Imitationsabsicht vorlag oder nicht.

Der Schematismus der Imitation, so kann man zusammenfassend sagen, steht nicht nur dem Nachahmer als Kontrollschema zur Verfügung, sondern insbesondere zweiten und dritten Parteien. Wenn es schließlich zutrifft, dass die Funktion der Imitation in der sozialen Orientierung liegt, kommt diesem Kontext, sei es der Urheber des Vorbildes selbst und/oder ein Publikum, mithin die wichtigste Rolle zu. Denn meistens gilt es, eben diesen Kontext zu beeindrucken, zumal aus dieser Richtung am ehesten jene semantischen Alarmsignale, Anreize und Sanktionen zu erwarten sind, von denen hier bereits die Rede war ${ }^{31}$. Eine Soziologie der Imitation wäre folglich als eine S o z i o l o g i e des bzw. der D ritten zu konzipieren. Die einfache Zurechnung der Imitation auf den Nachahmer gibt demgegenüber zwar die gängige Selbstbeschreibung der Imitation wieder, vermittelt jedoch, da sich die Praxis selbst nie ganz durchschaut und sich selbst simplifizieren muss, nur ein unvollständiges Bild, das in einer soziologischen

\footnotetext{
${ }^{30}$ MiCHAEL LEMPERT, Imitation, in: Annual Review of Anthropology 43, 2014, S. 379-395.

${ }^{31}$ Zum Zusammenhang zwischen Imitation und Belohnung/Bestrafung durch Dritte am Beispiel von Ratten und Kindern siehe HARTMUT ESSER, Situationslogik und Handeln (Soziologie. Spezielle Grundlagen 1), Frankfurt/Main 2002, S. 382-384.
} 
Fremdbeschreibung mit Begriffen wie ,Zurechnung' und ,Beobachtung' ergänzt werden müsste.

In der Sozialdimension, in der Konsens und Dissens sozial aufeinander bezogen werden, ist Imitation in mindestens zwei Hinsichten von Bedeutung: Sie ermöglicht nicht nur die Darstellung von Empathie, sondern involviert stets Dritte, die auf Versuche des Imitierens affirmativ oder ablehnend reagieren können. Mit Georg Simmel lässt sich sagen, „dass die Nachahmung eines der hauptsächlichen Mittel gegenseitigen Verständnisses ist; vermöge der vorhin betonten Association zwischen der äußeren Handlung und dem ihr $\mathrm{zu}$ grunde liegenden Bewußtseinsvorgang giebt uns die Nachahmung der Handlung eines andern oft erst den Schlüssel zu ihrem innerlichen Verständnis, indem die Gefühle, die früher auch bei uns die Handlung hervorriefen, erst durch jene psychologische Hülfe ihre Reproduktion erfahren." ${ }^{2}$ Insbesondere die zuvor erwähnte Möglichkeit des beinahe gleichzeitigen Imitierens kann, vor allem in der Interaktion unter Anwesenden, bereits auf einer körperlichen, vorsprachlich konstituierten Ebene zu einer ,emotionalen Stabilisierung'33 sozialer Systeme beitragenein Zusammenhang, der die Soziologie seit ihrer Gründerzeit vor allem am Beispiel von Massenbewegungen fasziniert hat ${ }^{34}$.

Neben dieser sozialen Schmiermittelfunktion ermöglicht Imitation ferner die Legitimation von Praktiken und Handlungen, die durch Verweis auf ein anerkanntes Vorbild mit der Unterstützung von Dritten rechnen können. Diese Position wird etwa weitgehend vom soziologischen Neo-Institutionalismus vertreten, der in der Imitation einen Mechanismus der Diffusion von Praktiken vermutet, der zu institutionellen Isomorphien führt, wie sie am Beispiel der globalen Verbreitung strukturell ähnlicher Nationalstaaten beobachtet werden können ${ }^{35}$. In dem Maße, in welchem legitime, also mit

\footnotetext{
${ }^{32}$ SiMMEL, Differenzierung (wie Anm. 18), S. 84. Aus Sicht der Systemtheorie müsste hier hinzugefügt werden, dass dieser Vorgang sozial nur dann relevant wird, wenn er in der Kommunikation als Bemühen um Empathie thematisiert wird. Damit schließt sie, anders als ihre Kritiker gelegentlich meinen, keineswegs die Möglichkeit gleichsinnigen Erlebens aus. Sie begründet diese Möglichkeit aber nicht anthropologisch oder subjekttheoretisch, sondern kann sie als Folge der Flüchtigkeit von Bewusstseins- und Kommunikationsereignissen, also von Gedanken und Handlungen, beschreiben. Gerade weil die Elemente des Bewusstseins und Kommunizierens gleichermaßen flüchtig sind, können sie mit hoher Wahrscheinlichkeit sinnhaft zusammenfallen. Das ändert nichts daran, dass Bewusstsein und Kommunikation mit unterschiedlichen Elementen operieren, die nicht füreinander substituierbar sind.

${ }^{33}$ Niklas LuhmanN, Funktionen und Folgen formaler Organisation, Berlin ${ }^{4} 1995$, S. 372-374; Siehe auch CHRISTIAN MEYER u. a., Intercorporeality. Emerging socialities in interaction, New York 2017.

${ }^{34}$ RoBert E. PARK, Masse und Publikum. Eine methodologische und soziologische Untersuchung, Bern 1904, S. 64-66; SiMMEL, Differenzierung (wie Anm. 18), S. 83-85.

${ }^{35}$ Paul J. Dimaggio - Walter W. Powell, The Iron Cage Revisited. Institutional Isomorphism and Collective Rationality in Organizational Fields, in: American Sociological Review 48, 1983, S. 147-160, hier S. 152-153
} 
kulturellen Werten vermeintlich übereinstimmende Praktiken übernommen werden, könne demnach ein sozialer Akteur mit der Unterstützung von Dritten rechnen und als Vorbild für weitere Nachahmer fungieren. Im Lichte der hier vorgeschlagenen Neufassung des Imitationsbegriffs lässt sich nun jedoch formulieren, dass ein Publikum Vorbilder konstruiert, um diese mit anderen Akteuren abzugleichen, nach Abweichungen (oder Entkopplungen) zu suchen, und erst auf Grundlage dieser Bewertung Unterstützung zu gewähren oder zu entziehen. Ein nach Legitimität strebender Akteur muss sich insofern nicht nur an einem Vorbild, sondern vor allem an den Konstruktionen und Vergleichsschemata eines Publikums orientieren ${ }^{36}$.

Imitation weist darüber hinaus polemogene Eigenschaften auf. Erste Ansatzpunkte liefert hierfür bekanntlich Rene Girards Theorie mimetischer Konflikte: Aufgrund der Orientierung auf dasselbe Objekt beobachten sich die Subjekte wechselseitig als Nachahmer und werden insbesondere dann, wenn das Objekt als knapp erlebt wird, zu Rivalen, die Konflikte mit Gewalt austragen und einander in ihrer Gewalttätigkeit imitieren, sofern dieser mimetische Teufelskreis nicht durch Opferung eines Sündenbocks durchbrochen werden kann ${ }^{37}$. Die imitatio nimmt dann agonale Züge an und die aemulatio folgt ihr auf schnellem Fuße. Damit ist zugleich gesagt, dass die soziale Funktion des Imitierens nicht in der Gewährleistung der Konformität des Verhaltens mit sozialen Erwartungen liegt, sondern in der Orientierung darüber, wie weit man voneinander abweichen muss, um sich auf dasselbe einlassen zu können, denn: „Abweichung und Dagegensein [ist] immer nur stark selektiv und auf Grundlage einer weitgehenden Übereinstimmung mit dem gesellschaftlichen Mainstream möglich [...]. Dies gilt auch dann, wenn in der Selbst- und Fremdwahrnehmung der relevanten Akteure das Element der Abweichung heraussticht und das Element der Übereinstimmung demgegenüber stark in den Hintergrund tritt.“38

\section{Analoge und äquivalente Alternativen zur Imitation}

Fasst man alle diese Leistungen zusammen, so kann man Vorteile der Zeit- und Kostenersparnis, der Orientierung in einer unsicheren Welt und der Legitimation eigener Handlungen hervorheben, denen gleichwohl problematische Aspekte der

\footnotetext{
$36 \mathrm{Zu}$ diesem Publikum kann, wie gesagt, auch das Vorbild selbst gehören, das mit missionarischem Sendungsbewusstsein eigene Strukturen als Modell empfiehlt.

${ }^{37}$ RENÉ GIRARD, Der Sündenbock, Zürich 1998; RENÉ GIRARD, Das Heilige und die Gewalt, Düsseldorf 2006.

${ }^{38}$ BORIS HOLZER - BARBARA KUCHLER, Globalisierungskritik und Weltkultur, in: IVONNE BEMERBURG - ARNE NiEDERBACHER (Hgg.), Die Globalisierung und ihre Kritik(er), Wiesbaden 2007, S. 75-93, hier S. 76.
} 
Konfliktanfälligkeit, der Identitätskrise und der rigiden Einschränkung von Vergleichshorizonten gegenüberstehen. Das legt die Suche nach Alternativen nahe, die der Imitation entweder oberflächlich ähneln oder ihre Funktion übernehmen können.

Eine gewisse Ähnlichkeit besteht zwischen dem Schematismus der Imitation und der Artikulation von Ansprüchen einerseits sowie dem Zweck/Mittel-Schema andererseits. Artikulierte Ansprüche ermöglichen analog zur Imitation ein Abtasten von sozialen Spielräumen und damit eine Orientierung in der Gesellschaft an Alarm- und Widerspruchssignalen. Wer Ansprüche auf eine optimale Gesundheitsversorgung, ein anspruchsvolles Fernsehprogramm und ideale Ehepartner erhebt, kontrolliert die eigenen Ansprüche mit deren Realisierung und wird in der gesellschaftlichen Umwelt auf Widerstände und Abwehrversuche gegen diese Ansprüche stoßen, die zu Anlässen des Aufbaus von Persönlichkeit und Ich-Stärke werden können ${ }^{39}$. Das Kriterium ertragbarer Frustration gewinnt dann an Orientierungswert, wenn man sich lernbereit zeigen und die eigenen Ansprüche auf ein realisierbares Niveau herunterfahren muss oder wenn man normativ auf eigenen Ansprüchen beharrt und dafür Unterstützung findet.

Das Zweck/Mittel-Schema weist ebenso Analogien mit dem Imitationsschema und der Artikulation von Ansprüchen auf. Dieses Schema vermittelt eine Orientierung in Entscheidungsprozessen, indem es Zwecke setzt und damit ein Spektrum einsetzbarer Mittel beobachtbar macht oder umgekehrt für bereits vorhandene Mittel adäquate Zwecke sucht. Es bietet Organisationen Kriterien für die Richtigkeit von Entscheidungen, die an Alarmsignalen wie Krisen, der Nicht-Erfüllung von Leistungsindikatoren oder dem laufenden Vergleich von Resultaten mit Organisationszwecken kontrolliert werden können 40 .

Obwohl Imitation, artikulierte Ansprüche und Zwecke ähnliche Muster aufweisen, lösen sie sehr unterschiedliche Probleme. Imitation bearbeitet das Problem der Abweichungskontrolle, Ansprüche beziehen sich auf Erwartungen in Leistungsbeziehungen und das Zweck/Mittel-Schema ist vor allem in (organisatorischen) Entscheidungsprozessen von Bedeutung. Das schließt Kombinationsmöglichkeiten keineswegs aus, wenn etwa Ansprüche imitiert werden oder eine Organisation in der Imitation ein Mittel für einen Zweck sieht. Statt aber diese Fragerichtung weiter zu

39 NIKLAS LUHMANN, Anspruchsinflation im Krankheitssystem. Eine Stellungnahme aus gesellschaftstheoretischer Sicht, in: JEANNE GUILLEMIN - PHILIPP HERDER-DorneICH (Hgg.), Die Anspruchsspirale. Schicksal oder Systemdefekt?, Stuttgart 1983, S. 28-49.

40 DERS., Zweckbegriff und Systemrationalität. Über die Funktion von Zwecken in sozialen Systemen, Frankfurt/Main ${ }^{2} 1977$, S. 322-324. 
verfolgen, gilt es nun, nach Alternativen zu suchen, die als Substitute für Imitation fungieren können, indem sie das Problem der Abweichungskontrolle mit anderen Mitteln lösen.

Zunächst können Praktiken und Semantiken der ,Singularisierung'41, oder umgekehrt formuliert: Verg le i chs - b zw. I m i t a ti o n s ve rb ote, als gegenläufige Substitute der Imitation verstanden werden. Was als einzigartig bzw. unnachahmlich gilt, wird auf diese Weise der Imitation entzogen, für die dann andere imitierbare Vorbilder gesucht werden müssen. Zu den wichtigsten Beispielen für derartige Verbote gehören die moderne Institution des Urheberrechts und ständische Imitationsverbote in vormodernen Gesellschaften, die sich etwa in schichtspezifischen Kleidungsvorschriften niederschlugen. Imitationsverbote sind außerdem im Bereich des Sakralen typisch, wo etwa heilige Schriften (man denke an den Koran), die Schöpfung und natürlich Gott selbst in die Transzendenz entrückt und als unantastbar und unnachahmlich definiert werden.

Ein weiteres Substitut für Imitation stellen L i m i t a ti o n e n dar. Schließlich fällt die Notwendigkeit einer Abweichungskontrolle dort weg, wo es ohnehin kaum Spielräume für Abweichungen und Variationen gibt. Das trifft etwa dann zu, wenn Einschränkungen des Lebensstils als Mangel an Kaufkraft erlebt und daher das Konsumverhalten einem ,Notwendigkeitsgeschmack ${ }^{42}$ (und nicht etwa an Moden oder unerreichbaren Vorbildern) folgen muss. In einem abstrakteren Sinne kann man außerdem von ökologischen oder sozialen Begrenzungen für soziale Problemlösungen sprechen. Einen besonderen aber nichtsdestotrotz verbreiteten Fall dafür bilden Phänomene der Konvergenz, also der Entwicklung ähnlicher Problemlösungen trotz unterschiedlicher Ausgangslagen ${ }^{43}$. So entwickelte sich der Gebrauch von Schriftzeichen mehrmals in voneinander unabhängigen Pfaden und konnte deshalb nicht von bereits beobachtbaren Vorbildern imitiert werden, sondern wurde zufällig und für ursprünglich ganz andere Probleme mnemotechnischer oder, wie in China, divinatorischer Art erfunden, von denen er sich später ablösen konnte ${ }^{44}$.

\footnotetext{
${ }^{41}$ ANDREAs RECKWITZ, Die Gesellschaft der Singularitäten. Zum Strukturwandel der Moderne, Berlin 2017.

${ }^{42}$ PIERRE BourdieU, Die feinen Unterschiede. Kritik der gesellschaftlichen Urteilskraft, Frankfurt/Main ${ }^{5} 1992$, S. 286.

${ }^{43}$ Alexander A. Goldenweiser, The Principle of limited Possibilities in the Development of Culture, in: The Journal of American Folklore 26, 1913, S. 259; zum Phänomen gleichzeitiger Doppelerfindungen s. ROBERT K. MERTON, The Sociology of Science. Theoretical and Empirical Investigations, Chicago ${ }^{2} 1974$, S. $343-382$.

${ }^{44}$ RUdOLF STÖBER, Innovation und Evolution. Wie erklärt sich medialer und kommunikativer Wandel?, in: CARSTEN Winter u. a. (Hgg.), Theorien der Kommunikations- und Medienwissenschaft, Wiesbaden 2008, S. 139-156, hier S. 145-153.
} 
Schließlich wären noch eine konsistente Selbstdarstellungsgeschichte bzw. ein erfolgreiches ,Stigma-Management'45 als funktionales Äquivalent der Imitation zu nennen. Die Imitation der Handlungen anderer erübrigt sich nämlich dann, wenn idiosynkratische Verhaltensabweichungen eine gewisse Erwartungssicherheit bieten, im sozialen Verkehr als Prämisse der Kontaktaufnahme berücksichtigt werden und ihre Ursachen zumindest in groben Zügen bekannt sind. Begegnet man in Wien einem stadtbekannten ,Original', so ist dessen Verhaltensabweichung immerhin erwartbar und wird eines gewissen Unterhaltungswertes wegen mitunter sogar geschätzt, sodass jenes Original von Imitations- und Konformitätserwartungen dispensiert werden kann. Dass umgekehrt auch die Imitation in diesem Sinne anerkannte Abweichungen substituieren kann, lässt sich insbesondere dann beobachten, wenn ein Fremder in einer neuen Umgebung als „Mensch ohne Geschichte“46 erlebt, und in seinem eigenen abweichenden Verhalten nicht unterstützt wird. Die eigene Selbstdarstellungsgeschichte kann er durch Imitation der Verhaltensweisen der neuen Umgebung ersetzen, indem er sich nach Möglichkeit so verhält, als ob er immer schon dazugehört hätte. Vergleichbares gilt, wenn das Christentum im Übergang von der Spätantike zum Mittelalter keine eigene vorzeigbare Texttradition aufweisen kann und diese durch Imitation heidnischer Klassiker substituiert ${ }^{47}$. Wo aber je gegenwärtige Imitation die Lücken einer eigenen problematischen Vergangenheit ausfüllt, müssen freilich die Brüche im Übergang von der eigenen zur angeeigneten Verhaltensweise kaschiert werden - sei es im einen Fall durch taktvolle Kommunikation, die es vermeidet, die dubiose Herkunft des Neulings zu thematisieren, der nun die eigenen Sitten und Bräuche mehr schlecht als recht imitiert ${ }^{48}$, sei es im anderen Fall durch Umdeutung einer heidnischen zu einer monotheistischen und damit: anschlussfähigen Tradition ${ }^{49}$.

Imitation scheint also im Lichte der genannten Alternativen kein unhintergehbarer Modus der Vergesellschaftung schlechthin $\mathrm{zu}$ sein. Sie ist, wie hier bereits wissenssoziologisch argumentiert wurde, auch keine anthropologische Konstante. Eine

\footnotetext{
${ }^{45}$ Erving Goffman, Stigma. Notes on the management of spoiled identity, Englewood Cliffs, N.J. 1963.

${ }^{46}$ Alfred SchÜtz, Der Fremde, in: Arvid Brodersen (Hg.), Gesammelte Aufsätze, Dordrecht 1972, S. 53-69, hier S. 60.

${ }^{47}$ Dina DE RentiIs, Art. Imitatio, in: Gert Ueding u. a. (Hgg.), Historisches Wörterbuch der Rhetorik, Bd. 4, Tübingen 1998, Sp. 235-303, hier Sp. 247.

${ }^{48}$ Verhältnismäßig taktlos würden vermutlich Autoren wie Augustinus argumentieren, der selbst die vollkommene Nachahmung christlicher Tugenden durch einen Heiden allenfalls als dessen hervorragendstes Laster bezeichnet, da allein die Nachahmung der Tugend keine christliche Substanz ersetzen kann.

49 John Marenbon, Pagans and Philosophers. The Problem of Paganism from Augustine to Leibniz, Princeton 2015.
} 
historische Soziologie kann daher für Vergleichszwecke das Bezugsproblem des Imitierens als Konstante setzen, um daraufhin variierende Bedingungen, Trends und Konjunkturen des Imitierens zu thematisieren.

\section{Desiderate einer historischen Soziologie der Imitation}

Wollte man in einer ersten Annäherung an die historische Karriere des Imitationsschemas einen langfristigen Trend ausfindig machen, könnte man von einem Führungswechsel zwischen einer Präferenz für Konformität und einer Präferenz für Abweichungen sprechen. Im Gegensatz zur Gesellschaft Alteuropas scheint gegenwärtig der Sinn der Imitation nicht darin zu liegen, ein möglichst perfektes Abbild eines Vorbildes zu gewährleisten bzw. dieses gar zu übertreffen, sondern Ansatzpunkte für jene Abweichungen von Vorbildern zu ermitteln, die als Indikatoren für die eigene Originalität und Individualität fungieren können. Dieser Führungswechsel ist aber nur ein Symptom viel grundlegenderer gesellschaftlicher Umbrüche, die sich als Übergang von der Anwesenheits- und schriftbasierten Gesellschaft zur Buchdruckgesellschaft, als Übergang von der stratifizierten Gesellschaft Alteuropas zur funktional differenzierten Moderne oder als Übergang vom Weltbild eines geordneten Kosmos hin zur Vorstellung von der Welt als einem Horizont von Möglichkeiten charakterisieren lassen ${ }^{50}$. Statt eine erschöpfende Darstellung dieser Zusammenhänge anzubieten, können hier vorläufig nur Fragen aufgeworfen werden, die Desiderate künftiger Forschung markieren sollen.

Dass die Einführung der Schrift die Beobachtung und Thematisierung von Imitation überhaupt erst nahelegt, wurde bereits angesprochen. Was geschieht jedoch, wenn durch Technologien des Buchdrucks und der Massenmedien die Funktion der Abweichungskontrolle nicht mehr von einer überschaubaren Kaste von Priestern und Schriftgelehrten erfüllt werden kann, die Autoren zu nachzuahmenden Autoritäten und bewahrenswerte Texte zu vorbildlichen kanonischen Texten deklarieren ${ }^{51}$, sondern z. B. von Rankings übernommen wird, die nun Vorbilder definieren und den Abstand von diesen Vorbildern durch Quantifizierung messen ${ }^{52}$ ? Welche Folgen haben Massenmedien ferner, wenn elementare Verhaltensweisen wie etwa die Anbahnung intimer Kontakte einer Vielzahl von Medienkonsumenten vorgeführt werden, die aus dem Gesehenen und

\footnotetext{
${ }^{50}$ LUHMANN, Die Gesellschaft (wie Anm. 14), S. 707-709.

${ }^{51}$ ARIE VAN DER KoOIJ u. a. (Hgg.), Canonization and decanonization, Leiden 1998.

52 RAMY YousSEF, Rankings statt Stellenordnungen? Funktionen und Folgen von Leistungsvergleichen in internationalen Organisationen, in: CHRISTOPHER DORN - VERONIKA TACKE (Hgg.), Vergleich und Leistung in der funktional differenzierten Gesellschaft, Wiesbaden 2018.
} 
Gelesenen auf sich selbst schließen und abwesende fiktive Vorbilder nachahmen, statt den schmerzvollen Weg des ,Trial-and-Error' zu beschreiten oder sich an realen Vorbildern zu orientieren? Bereits Don Quijotes klägliche Versuche in Rittertum und Minnedienst führen die Folgeprobleme einer mimetischen Identifikation mit fiktiven und abwesenden Vorbildern eindrücklich vor Augen. Außerdem stellt sich die Frage, wie es dazu kommt, dass gegenwärtig Individualität, Originalität und Kreativität zelebriert werden ${ }^{53}$, obwohl Massenmedien eine ungeheure Bandbreite möglichen Verhaltens bereits vorexerziert haben und Individualität paradoxerweise allenfalls in der Form einer ,kopierten Existenz' ausgelebt werden kann54? Mit zunehmender massenmedialer Kommunikation gewinnt nicht zuletzt auch die Werbung an Bedeutung, die ständische Konsumgebote und Imitationsverbote in ihrer Funktion der Orientierung des Konsumverhaltens ersetzt. Die Entstehung von Moden lässt sich dann nicht mehr hinreichend als Imitation der Oberschicht durch die Unterschicht interpretieren, sodass die Oberschicht dazu angehalten wäre, ständig neue Moden zu entwickeln ${ }^{55}$. Vielmehr wird selbst das Konsumverhalten der Oberschicht am „Geschmacksdiktat der Werbung“56 und an Preissignalen orientiert.

In einem allgemeineren Sinne hätte eine historische Soziologie der Imitation jedenfalls zu klären, wie die Ausdifferenzierung von gesellschaftlichen Teilbereichen wie Wirtschaft, Wissenschaft, Politik etc. und damit einhergehend die Ausdifferenzierung von Kommunikationsmedien wie Geld, Wahrheit und Macht durch Imitation zu beschreiben wäre. Das bezieht sich zunächst auf Prozesse der Expansion dieser Systeme im globalen Maßstab, die im Endergebnis zur Entstehung einer einzigen Weltgesellschaft geführt haben. Imitation dürfte dabei je nach gesellschaftlichem Bereich von unterschiedlicher Bedeutung gewesen sein: In Kunst und Wissenschaft muss Imitation gegenüber besonderen Ansprüchen an Originalität begründet werden, die man etwa im Sport oder in der Politik nicht antreffen wird, wo die offenkundige Orientierung an Vorbildern viel unproblematischer zu sein scheint - solange nur das Vorbild plausibel und vertretbar ist.

\footnotetext{
53 ANDREAS ReCKWITZ, Die Erfindung der Kreativität. Zum Prozess gesellschaftlicher Ästhetisierung, Berlin 2012; DERS., Kreativität und soziale Praxis. Studien zur Sozial- und Gesellschaftstheorie, Bielefeld 2016.

${ }^{54}$ NiKLAS LuHMANN, Copierte Existenz und Karriere. Zur Herstellung von Individualität, in: UlRICH BECK (Hg.), Riskante Freiheiten. Individualisierung in modernen Gesellschaften, Frankfurt/Main ${ }^{2}$ 1994, S. 191-200; DERS., Individuum, Individualität, Individualismus, in: DERS., Gesellschaftsstruktur und Semantik 3. Studien zur Wissenssoziologie der modernen Gesellschaft, Frankfurt/Main 1993, S. 149-258.

${ }^{55}$ Für eine Kritik dieser These s. ElENA EsPosito, Die Verbindlichkeit des Vorübergehenden. Paradoxien der Mode, Frankfurt/Main 2004, S. 19-21.

${ }^{56}$ Niklas LuhmanN, Die Realität der Massenmedien, Wiesbaden ${ }^{2} 1996$, S. 90.
} 
Schließlich können Zusammenhänge zwischen historisch variierenden Semantiken der Imitation und Umbrüchen der Weltkonzeption im Übergang zwischen der Gesellschaft Alteuropas und der modernen Gesellschaft thematisiert werden. Während sich der alteuropäische Begriff der Imitation primär auf die Vorstellung eines geordneten Kosmos bezog, der alle nachzuahmenden Formen, Arten und Gattungen in sich enthielt und damit die menschliche Erschaffung neuer Formen ausschloss ${ }^{57}$ - sei es, weil die Schöpfung Gott vorbehalten war, oder, wie in der griechischen Tradition, eine creatio ex nihilo ohnehin unvorstellbar war58 -, formiert sich im Übergang zur modernen Gesellschaft der Begriff der Innovation explizit als Gegenmodell zur Imitation. Dem Anspruch, dem Wesen einer idealen Form durch Nachahmung gerecht zu werden, folgt zunächst in den ästhetischen Reflexionstheorien einer sich ausdifferenzierenden Kunst der Anspruch, neue Formen nicht zu finden, sondern zu er-finden ${ }^{59}$. Spätestens wenn diese Semantik der Originalität Strukturwert gewinnt, kann nicht mehr von einem prästabilisierten Kosmos ausgegangen werden, der alle Formen vorgibt. Stattdessen wird die Gattungsordnung aufgesprengt in einen Horizont, der nun nicht zwischen einer momenthaften weltlichen Zeit der Imitation (tempus, kairos) und einer ewigen Gegenwart der Vorbilder (aeternitas, chronos bzw. aion) unterscheidet, sondern zwischen den Horizonten der Vergangenheit und der Zukunft, wobei letzterer zunehmend die Führung im Erleben und Handeln gewinnt und in der Gegenwart allenfalls noch Anhaltspunkte aus der Vergangenheit herangezogen werden müssen, um künftige Tendenzen und Risiken abschätzen zu können. Imitation bedeutet dann folglich, sich gegenwärtig an der Vergangenheit zu orientieren, um künftige Folgen antizipieren zu können, sodass sich selbst Reflexionstheorien der Moral nicht mehr auf zeitlose Vorbilder beziehen und die imitatio Christi empfehlen können, sondern nun aufgefordert sind, ethische Leitlinien primär mit Blick auf nichtabsehbare Handlungs- und Technikfolgen zu formulieren ${ }^{60}$. Im

\footnotetext{
${ }^{57}$ Auch der Begriff der poiesis meinte zunächst keine schöpferische Tätigkeit des Menschen im Sinne eines Erfindens neuer Formen, sondern bezog sich auf die Orientierung einer Tätigkeit an einem äußeren Zweck.

58 Max Weber vermutete einen Zusammenhang zwischen den Herrschaftsstrukturen der hydraulischen Gesellschaften Vorderasiens und der Vorstellung eines Gottes, der die Welt aus dem Nichts erschaffen hat, s. WEBER, Wirtschaft (wie Anm. 12), S. 255-256. Die politischen Strukturen der griechisch-römischen Antike hatten im Gegensatz dazu offenbar keinen Bedarf an derartigen Schöpferfiguren. Selbst die ziemlich opake Figur des platonischen Demiurgen, der dieser Vorstellung eines Schöpfergottes wohl noch am nächsten kommt, setzt die immer schon vorliegenden Ideen im Kosmos lediglich um und zieht sich daraufhin aus dem Weltgeschehen zurück.

${ }^{59}$ CORNelia BoHn, Schriftlichkeit (wie Anm. 14), S. 202-204; Alois HaHN, Kunst, Wahrnehmung und Sinndeutung, in: DERS., (Hg.), Konstruktionen des Selbst, der Welt und der Geschichte. Aufsätze zur Kultursoziologie, Frankfurt/Main 2000, S. 407-439, hier S. 435-437; NIKLAS LuHMANN, Die Kunst der Gesellschaft, Frankfurt/Main 1995, S. 424-426.

${ }^{60}$ GeOrg Pfleiderer - ChristoPh ReHMANN-Sutter (Hgg.), Zeithorizonte des Ethischen. Zur Bedeutung der Temporalität in der Fundamental- und Bioethik, Stuttgart 2006.
} 
Gegensatz zur alteuropäischen Kosmologie fordert der Doppelhorizont von Vergangenheit und Zukunft aber nicht bloß anderen, sondern auch ständig neuen Sinn ein und man geht dazu über, novitas nicht mehr primär als sachliche Abweichungen vom Gewohnten, gleichsam als eine Sonderform der varietas zu begreifen ${ }^{61}$, sondern als eine Zeitfolge, die bereits als solche Neuheiten rechtfertigt und begrüßenswert erscheinen lässt ${ }^{62}$. Statt der aemulatio, also dem gegenwärtigen Wetteifern um größtmögliche Annäherung an ein ewiges Gattungsideal, wird im Verlauf der Frühen Neuzeit die neuzeitliche Semantik der Konkurren ${ }^{63}$ um das Finden neuer Formen zu einem Leitmotiv in Wirtschaft, Wissenschaft und Kunst. Gerade der Begriff der Imitation erfährt in dieser Umbruchszeit enormen Aufschwung, weil er sowohl für die Orientierung an einer kanonisierten Tradition als auch für das Prinzip der Erfindung durch die Kombination bewährter Formen stehen kann. Überhaupt scheint er geradezu eine Krisenund Abwehrsemantik darzustellen, die immer dann prävalent wird, wenn die bestehende Ordnung der Welt gefährdet zu sein scheint und Imitation als Rezept zur Erhaltung dieser Ordnung empfohlen wird ${ }^{64}$. Eine primäre Aufgabe der historischen Wissenssoziologie müsste folglich darin bestehen, diesen Krisen und Konjunkturen nachzuspüren und nach Zusammenhängen mit gesellschaftsstrukturellen Umbrüchen zu fragen.

Die hier angestellten Überlegungen deuten darauf hin, dass weder die gesellschaftliche Differenzierungsform noch verfügbare Verbreitungstechnologien oder der Wandel von Weltbildern jeweils allein ausschlaggebend für historische Konjunkturen der Imitation sind, sondern dass sie einander bedingen und auf unterschiedliche Art und Weise mit Semantiken der Imitation korrelieren. Dabei wurden Zusammenhänge zwischen Imitation einerseits und Sozialisation, sozialem Wandel und der Verbreitung und Diffusion von Wissen andererseits noch gar nicht berührt. Nur so viel scheint vorläufig auf der Hand zu liegen: dass Imitation (im Gegensatz zu ihrem Bezugsproblem!) kein ahistorischer Sachverhalt ist und mit Sicherheit auch nicht jenen singulären Vergesellschaftungsmechanismus darstellt, der das Soziale schlechthin konstituiert.

\footnotetext{
${ }^{61}$ ALEXANDRU CIZEK, Imitatio et tractatio. Die literarisch-theoretischen Grundlagen der Nachahmung in Antike und Mittelalter, Berlin 1994, S. 77-78.

62 Niklas LuHMANN, Die Behandlung von Irritationen. Abweichung oder Neuheit? in: DERS., Gesellschaftsstruktur und Semantik. Studien zur Wissenssoziologie der modernen Gesellschaft, Bd. 4, Frankfurt/Main ${ }^{1}$ 1999, S. 55-100; ElENA EsPOSITO, Die Verbindlichkeit (wie Anm. 55), S. 96-113.

${ }^{63}$ Tobias Werron, Konkurrenz als historischer Begriff, in: Ralf JeSSEN (Hg.), Konkurrenz in der Geschichte, Frankfurt/Main 2014, S. 59-94.

${ }^{64}$ Rudolf STICHWEH, Der frühmoderne Staat und die europäische Universität. Zur Interaktion von Politik und Erziehungssystem im Prozess ihrer Ausdifferenzierung (16.-18. Jahrhundert), Frankfurt/Main 1991, S. $133-142$.
} 
Umso wichtiger wird es für eine künftige Wissenssoziologie der Imitation deshalb sein, ihren Gegenstand historisch zu kontextualisieren und dafür Kontakte - und womöglich auch Vorbilder - in der Geschichtswissenschaft zu suchen. Umgekehrt mag vielleicht eine Soziologie nach dem Vorbild Niklas Luhmanns für geschichtswissenschaftliche Fragestellungen instruktiv sein. Wie viel Konformität und wie viel Abweichung von diesem Vorbild nötig sein werden, um dem Gegenstand der Imitation gerecht zu werden, muss jedoch dem Urteil einer Fachöffentlichkeit überlassen bleiben. 